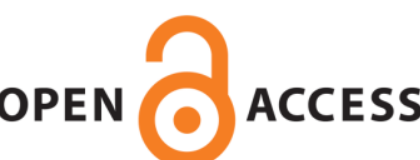

OPEN ACCESS

UWS Academic Portal

\title{
Strength adaptation to squat exercise is different between Caucasian and South Asian novice exercisers
}

Knox, Allan; Sculthorpe, Nicholas; Baker, Julien S.; Grace, Fergal

Published in:

Research in Sports Medicine

DOI:

$10.1080 / 15438627.2017 .1314293$

E-pub ahead of print: 16/04/2017

Document Version

Peer reviewed version

Link to publication on the UWS Academic Portal

Citation for published version (APA):

Knox, A., Sculthorpe, N., Baker, J. S., \& Grace, F. (2017). Strength adaptation to squat exercise is different between Caucasian and South Asian novice exercisers. Research in Sports Medicine, 25(3), 373-383.

https://doi.org/10.1080/15438627.2017.1314293

\section{General rights}

Copyright and moral rights for the publications made accessible in the UWS Academic Portal are retained by the authors and/or other copyright owners and it is a condition of accessing publications that users recognise and abide by the legal requirements associated with these rights. 


\title{
Title
}

Strength Adaptation to Squat Exercise is Different Between Caucasian and South Asian Novice Exercisers.

\begin{abstract}
This study compared the progression of muscular strength (MS) adaptation between agematched Caucasian (CAUC) and South Asian (SOU) men during 6 weeks $\left(3 \mathrm{x} \mathrm{week}^{-1}\right)$ of resistance training. MS was determined pre and post intervention by 3-repetition maximum (3RM) strength tests, and data were analysed using repeated measures ANOVA. Preintervention upper and lower body 3RM were similar between groups and both upper and lower body 3RM increased in CAUCs $(P<0.001)$ and SOUs $(P<0.001)$ following resistance training. However, lower body strength adaptation (3RM) was higher in CAUCs compared with SOUs $(P=0.002)$. There was a significant group $\mathrm{x}$ time interaction in strength progression of the squat exercise $(P=0.03)$ from session 7 through to 18 (completion). The present study offers novel but provisional data that lower body strength adaptation is slower in SOU than CAUC men despite comparable adaptation to upper body strength.
\end{abstract}

Key words: Resistance Training; Racial; South Asian; Regional; Adaptation. 


\section{Introduction}

Racially specific determinants of athletic performance has received a small amount of previous attention in the academic literature (Ama et al., 1990; Coetzer et al., 1993; Swift et al., 2013). In attempting to identify racial characteristics of this exercise response, elite athletic performers have been a topic of episodic public and scientific interest during the past three decades. For instance, Black East African distance runners and Black sprinters of West African and Caribbean origin have dominated endurance and sprint events in World athletics, respectively. These observations prompted investigation and subsequent identification of polymorphisms such as the $\alpha$-actinin-3 R577X that demonstrate consistent associations with elite power and sprinting performances (Eynon et al., 2013). Although these endeavours have, to some degree, progressed our understanding of elite performance, the over-arching concept that muscular adaptation to exercise training may differ between racial groups, remains largely overlooked. Apart from a few small studies (Hall et al., 2010; Cubbon et al., 2010; Knox et al., 2016), South Asian (SOU) ex-patriates are largely unrepresented in the exercise science literature. SOU's originate from locations surrounding the Indian subcontinent which include countries such as India, Pakistan, Bangladesh and Sri Lanka. SOU expatriates are the largest and fastest growing ethnic minority in Western Society and account for $20 \%$ of the world's population (CelisMorales et al., 2013). Recently, a novel cross-sectional study identified that SOU men may need to engage in 266 minutes.week ${ }^{-1}$ of moderate intensity aerobic exercise to generate equivalent cardio-metabolic responses with Caucasian (CAUC) men undertaking the recommended guidelines of 150 minutes. week $^{-1}$ (Celis-Morales et al., 2013), suggestive of a blunted response to magnitude of exercise-training induced cardio-metabolic improvement in SOUs compared with CAUCs.

The few available exercise training intervention studies have been largely dedicated to comparison of African Americans with CAUC Americans, predominantly using clinical and 
aerobic fitness outcome measures (Cook et al., 2013; Fields et al., 1997; Walts et al., 2008; Coetzer et al., 1993). Data on muscular adaptation to resistance exercise in different racial groups is lacking. Specifically, there are no studies that compare strength adaptations to resistance exercise in SOU's with other racial cohorts. Such data may provide evidence that racial background may have to be considered by exercise practitioners when programming resistance exercise.

Therefore, the aim of the present study was to compare adaptations to a 6-week resistance training programme in SOU's compared with a group of aged matched CAUCs. The short-term protocol is necessary to establish training adaptations independent of skeletal muscle hypertrophy. This would suggest that any observed adaptations were predominantly neuromuscular induced changes. Conversely, studies of muscular adaptation to resistance training have failed to find differences in the hypertrophic response between races. Walts et al. (2008) reported that resistance training produced equivalent hypertrophy in Caucasian and African American men and women. We hypothesised that strength adaptation, determined by upper and lower body strength tests, in response to a 6-week progressive resistance training programme, would be different in SOU's compared with a control group of age matched CAUC males. 


\section{Methods}

\section{Participants}

A total of 19 CAUC and 19 SOU male volunteers provided written informed consent to participate which was approved by University of the West of Scotland ethics committee. Each subject fully understood the risks and benefits involved with participation. Prior to enrolment, subjects did not engage in any recreational or competitive sporting activities, nor did they have any prior experience of resistance training, which was confirmed by physical activity questionnaire. Fifteen CAUCs (25.5 \pm 4.8 years) and 13 SOUs $(25.4 \pm 7.0$ years $)$ adhered to $100 \%$ of training sessions and were included in the final analysis.

\section{Anthropometrics}

Height was determined using a portable stadiometer (Leicester Height Measure, Seca, Birmingham, U.K.). Body mass was measured to the nearest $0.1 \mathrm{~kg}$ by commercially available scales (body composition analyser TBF-300, Tanita, Tokyo, Japan). Body mass index was calculated using the following formula; BMI = body mass $(\mathrm{kg}) \div$ height $(\mathrm{m})^{2}$. Total body fat percentage was calculated using bioelectrical impedance analysis (BIA) using a commercially available analyser (body composition analyser TBF-300, Tanita, Tokyo, Japan).

\section{Muscular Strength Measures}

Upper and lower body MS was measured by 3-repetition max (3RM) bench press and squats respectively, using a commercially available 20kg Olympic barbell (Pendlay Nexgen, Fort Mill, USA), Olympic weighted plates of various weight including $25 \mathrm{~kg}, 20 \mathrm{~kg}, 15 \mathrm{~kg}, 10 \mathrm{~kg}$, $5 \mathrm{~kg}, 2.5 \mathrm{~kg}, 1.25 \mathrm{~kg}$ (Taishan Sports Industry Group, Burlingame, USA), a standard power cage and bench press. Prior to the first attempt of the test, each participant was instructed to complete a warm-up with light resistance which would allow for 10-15 repetitions. Following a 1 minute 
rest period, a load would then be applied which enabled the participant to perform 5-10 repetitions. A further rest period of 2 minutes was provided. The load was further increased which would allow for the participant to perform 4-8 repetitions. Five minutes' rest period followed which concluded the warm up procedure.

The load was increased to an estimate of $3 \mathrm{RM}$ judging from previous sets in the warm up. The participants were then instructed to perform a 3RM. A successful attempt was defined as all repetitions completed without any deterioration in technique, with a failed attempt defined as not achieving the required repetitions or executing poor technique. If successful, then a 5minute rest period was provided before the load was increased. An attempt of a higher load would consequently result. If the participant failed at a higher load, then the previous attempt would be recorded as the final result. The final load in which the participant was successful at 3RM was recorded and used for analysis. These protocols were performed at baseline (PRE) and following resistance training (POST). The 3RM test was performed 3 days following the final training session. This was standardised in both groups.

\section{Progressive Resistance Training Protocol}

The resistance exercise prescription followed a linear progression model involving five compound exercises; including back squats, bench press, deadlifts, shoulder press, and lateral pull down, with additional accessory exercises. These exercises were separated into two different sessions; A and B (see Table 1). Each session was performed consecutively within the three training days per week (i.e. week 1; A, B, A, week 2; B, A, B, etc.) for the six-week duration of the study. Training days were separated by at least one rest day but no longer than two rest days.

Due to the inexperience of the participants in resistance training, it was essential that training loads were prescribed to ensure correct technique was performed. The initial intensity of 
training is presented in Table 2. This ensured each participant was capable of performing each exercise safely. Each subject was required to complete three sets of ten repetitions, which has been shown to significantly increase the desired adaptations of resistance training of a novice participant (ACSM, 2009).

The criteria for training progression was the completion of the required sets and repetitions ( 3 x 10), without any deterioration in technique for any given exercise. Progressive loads for lower body exercises (squats and deadlifts) were standardised at $5 \mathrm{~kg}$ increases, whereas upper body exercises (shoulder press, lateral pull down, bench press, abdominal crunches, barbell rows) progressed by $2.5 \mathrm{~kg}$.

\section{Statistical Analysis}

Data were analysed using SPSS version 22. Group and time interactions, main effects of time and group, simple main effects of time and group were determined by a mixed model ANOVA with repeated measures. Distribution of data were assessed by Shapiro-Wilk tests. Homogeneity of variances was assessed by Levene's test of homogeneity of variance. Homogeneity of co-variances was established by Box's test of equality covariance matrices. All assumptions were not violated $(P>0.05)$ unless otherwise stated. Where Mauchly's test of Sphericity was violated $(P<0.05)$, the Greenhouse-Geisser estimate is reported. An alpha value of $P<0.05$ was used to indicate statistical significance. Accumulated loads during each exercise session, anthropometric and 3RM data were analysed PRE and POST intervention using a $2 X 2$ mixed model ANOVA, which are presented as $P$ value and effect size $\left(\eta^{2}\right)$. Partial eta-squared values were used to estimate effect size in the presence of statistically significant differences. Bonferroni corrected independent t-tests were employed for post-hoc multiple comparisons. (Exemplar: where significant interaction was evident during the progression of squat exercise (consisting 18 sessions), statistical significance was set at $P<0.003$, to account for multiple comparisons in sessions 7-18). Cohen (1988) guidelines, where $0.2=$ small effect, 
$0.5=$ moderate effect, and $0.8=$ large effect, were used to interpret effect size between 2 groups. Post hoc data are presented as mean difference $(M), 95 \%$ CI, $P$ value. Statistical significance was set at $P<0.05$. Data is presented as group mean \pm standard deviation (S.D.). 


\section{Results}

PRE and POST characteristics can be found in Table 3.

\section{Anthropometrics}

No significant group $\times$ time interactions, main effects of time or group were observed following resistance training in any measure $(P>0.05$ for all measures).

\section{Muscular Strength}

Figure 1 shows a significant group $\times$ time interaction in lower body strength following resistance training $\left(P=0.002\right.$, partial $\left.\eta^{2}=0.302\right)$. There was a significant difference at POST between groups $\left(P=0.013\right.$, partial $\left.\eta^{2}=0.213\right)$ with the CAUC presenting significantly greater lower body strength than the SOU group $(M=23.462 \mathrm{Kg}$, $95 \% \mathrm{CI}: 5.278$ to $41.645 \mathrm{Kg}, P=$ 0.013). A significant simple main effect of time was observed in the CAUC group $(P<0.001$, partial $\left.\eta^{2}=0.910\right)$ and the SOU group $\left(P<0.001\right.$, partial $\left.\eta^{2}=0.809\right)$ at POST.

There was no significant group $\times$ time interaction between following resistance training on upper body strength $\left(P=0.476\right.$, partial $\left.\eta^{2}=0.020\right)$. The CAUC group $(M=13.667 \mathrm{Kg}, 95 \%$ CI: 10.293 to $17.040, P<0.001)$ and SOU group $(M=11.923 \mathrm{Kg}, 95 \%$ CI: 8.3 to $15.546, P<$ 0.001) had significantly improved upper body muscle strength at POST. 


\section{$1 \quad$ Resistance Exercises}

2 There was a significant group $\times$ time interaction on the progression on squats $(P<0.001$, partial

$3 \eta^{2}=0.282$ ). Figure 2 shows significant differences in squat progression between groups 4 observed from session 7 which continued until the end of the intervention $(P<0.05$ for all time 5 points).

6 Significant increases in bench press $(M=14.167 \mathrm{Kg}, 95 \% \mathrm{CI}: 8.691$ to $19.462 \mathrm{Kg}, P<0.001)$, 7 deadlifts $(M=36.667,95 \%$ CI: 28.103 to $45.230 \mathrm{Kg}, P<0.001)$, shoulder press $(M=13.667$, 95\% CI: 9.552 to $17.781 \mathrm{Kg}, P<0.001)$ and lateral pull down $(M=18.333 \mathrm{Kg}, 95 \% \mathrm{CI}: 15.117$ to $21.550 \mathrm{Kg}, P<0.001)$ were observed in the CAUC group at POST.

10 The SOU group experienced significant increases in bench press $(M=13.269 \mathrm{Kg}$, $95 \% \mathrm{CI}$ : 7.387 to $19.151 \mathrm{Kg}, P<0.001)$, deadlifts $(M=33.077 \mathrm{Kg}, 95 \%$ CI: 23.646 to $45.508 \mathrm{Kg}, P<$ 0.001), shoulder press $(M=14.231 \mathrm{Kg}, 95 \% \mathrm{CI}$ : 9.811 to $18.651 \mathrm{Kg}, P<0.001)$ and lateral pull down $(M=18.462 \mathrm{Kg}, 95 \% \mathrm{CI}: 15.007$ to $21.916 \mathrm{Kg}, P<0.001)$ at POST.

\section{Discussion}

16

The aim of this study was to compare muscular strength adaptations between CAUC and SOU men, during 6-weeks of resistance training. The main findings from this study are that 6-weeks of progressive resistance training improves upper and lower body MS in both CAUC and SOU males, however, lower body MS adaptation was slower in SOU compared with CAUC, independent of changes in body composition. These data provide preliminary evidence for racial differences in lower limb strength adaptation to resistance exercise between SOU and CAUC men.

The observed findings in the current study show that the rate of adaptation between racial groups is comparable in terms of upper body adaptation, but is blunted with respect to lower body training adaption in SOUs. One possible explanation for the observed difference may be 
related to a difference in neural adaptation in response to resistance training. Significant increases in muscular strength are observed independent of increases in muscular hypertrophy during 3-5 weeks of resistance training which is mainly accounted for by an improvement in neural recruitment (Cormie et al., 2011; Folland \& Williams, 2007). Furthermore, the ability to generate muscular power in order to execute a movement under a mechanical load is influenced by a variety of factors including; muscle fibre composition, cross-sectional area, motor unit recruitment, and inter-muscular coordination. Since the lower body 3RM test requires a larger degree of motor unit recruitment and inter-muscular coordination to execute (compared to the upper body protocol), it may be that differences between SOU and CAUC where more evident during this assessment. However, it is difficult to confirm these findings given the lack of comparable data concerning training responses in SOU cohorts. Previous cross-sectional research reported no differences in power outputs between African Americans and CAUCs when corrected for muscle cross-sectional area (Fields et al, 1997). Similarly, a longitudinal study also reported no racial differences between African Americans and CAUCs in knee extensor 1RM values following 10 weeks of localised knee extensor resistance training (Walts et al, 2008). Correspondingly, the higher rate of lower body adaption in the present study may be due to methodological factors in addition to the racial differences of the respective cohorts. The current study used whole body resistance training whereas Walts and associates (2008) concentrated on the effects of training on a single muscle group. Furthermore, the squat is a more complex compound movement than knee extensions, requiring significantly greater coordination, and therefore may better identify differences in neuromuscular adaptation. In addition, Walts et al. (2008) used a longer training programme which may have combined both neuromuscular and hypertrophic adaptation.

There is a scarcity of data comparing strength adaptations to resistance exercise performances 
strength in SOU's. It has been shown that BMI matched African Americans produce more favourable upper body strength than White Americans following 6-weeks resistance training (Cook et al., 2013). The authors reported greater upper body strength in the African American group at study enrolment, which may account for the differences in strength following resistance training, as no significant interactions were discovered (Cook et al., 2013). This also suggests that the rate of adaptation between the African Americans and White Americans were similar. The comparable results between the current study and that of Cook and co-workers (2013) may possibly relate to muscle group under investigation. The current investigation did not identify any significant interactions between groups in upper body strength following resistance training, which is in agreement with previous research (Cook et al., 2013). Taken in context with the available literature, it appears that racial differences in strength adaptation to resistance exercise may be confined to the lower limbs.

This is the first study to compare resistance training adaptions between CAUCs and SOUs. To borrow from the wider literature, Misra and colleagues (2008) reported improvements in insulin sensitivity in SOUs diagnosed with type 2 diabetes following 12 weeks of moderate intensity resistance training. However, details of the improvements in muscular strength were not reported, making it impossible to compare adaptations of muscular strength with the current investigation. More recent research has reported significant time effects in SOUs participating in resistance training (Hameed et al., 2012). The participants of this study performed progressive resistance training 2-3 times per week for 8 weeks performing similar exercises and rest periods as the current study. The authors reported significant increases in upper and lower body strength, (assessed by 1-RM bench press and leg press, respectively) when compared to a control group who performed static stretching for the duration of the intervention. The results of the current study and that of Hameed et al (2012) are in agreement that SOUs do adapt to progressive resistance training with increases in muscular strength. 
77 However, the current study postulates a theory that the magnitude of adaptation may be distinctive between racial groups.

It is well known that exercise is fundamental in the prevention of non-communicable diseases (NCDs) such as type 2 diabetes and cardiovascular diseases. The aim of current exercise guidelines is to reduce physical inactivity levels and consequent NCD risk (ACSM, 2009). This has resulted in general prescription guidelines for all age groups. However, in certain circumstances, more specific exercise guidelines may be required. SOUs have inferior prognosis following a cardiac event compared to CAUCs for unknown reasons, and structured exercise participation is a vital process in the final stages of cardiac rehabilitation programmes (Astin et al., 2008). Previous research regarding differences between CAUCs and SOUs response to exercise have only investigated aerobic exercise. Hall and associates (2010) reported that SOUs have a reduced capacity to oxidise fat during submaximal aerobic exercise compared to CAUCs of similar age, BMI and fat mass. Cubbon et al. (2010) also reported differences to aerobic exercise between CAUCs and SOUs, as exercise induced circulating progenitor cell mobilisation is reduced in SOUs compared to CAUCs. The work of CelisMorales (2013), show that SOUs may need to engage in 266 minutes of moderate intensity exercise to elicit similar adaptations as CAUCs performing 150 minutes, suggesting a blunted response to exercise. Although the current study has mainly studied differences in performance parameters of muscular strength and not clinical markers, it agrees with the existing literature that there are racially dependent responses to an exercise stimulus.

\section{Study Limitations}

The current study has identified differences in regional strength adaptations, which we propose to be consequence of differences in neuromuscular adaptation between CAUCs and SOUs. The significant increases in strength, independent of changes in body composition, provide evidence of neural adaptations in both groups following the short-term protocol. However, this 
102 remains speculative until verified by further electromyography (EMG) or electroneurography

103 (EMNG) study.

104 The short duration of the study may also pose as a potential limitation. The 6-week protocol

105 was sufficient to identify potential differences in neuromuscular adaptation however, a longer

106 protocol may have uncovered potential differences in hypertrophic response (Moritani \&

107 deVries, 1979). Whether the SOU group could match the rate of progression following a

108 hypertrophic specific programme remains to be investigated. It was impractical and without $a$

109 priori rationale to biopsy muscle in order to determine muscle fibre composition in this study.

110 Differences in muscle fibre type and distribution might explain the differences in regional

111 adaptations to resistance training as previous reports have identified muscle fibre composition

112 discrepancies between racial groups.

113 In conclusion, this study offers novel but provisional data that progression of lower body

114 strength adaptation is slower in SOU and CAUC men despite comparable adaptation to upper

115 body strength. These data may be used to inform exercise prescription and adaptive training

116 programmes for SOU men. 


\section{References}

ACSM 2009. American College of Sports Medicine position stand. Progression models in resistance training for healthy adults. Med Sci Sports Exerc, 41, (3), 687-708.

Ama, P. F., Lagasse, P., Bouchard, C. \& Simoneau, J. A. 1990. Anaerobic performances in black and white subjects. Med Sci Sports Exerc, 22, (4), 508-11.

Astin, F., Atkin, K. \& Darr, A. 2008. Family support and cardiac rehabilitation: a comparative study of the experiences of South Asian and White-European patients and their carer's living in the United Kingdom. Eur J Cardiovasc Nurs, 7, (1), 43-51.

Celis-Morales, C. A., Ghouri, N., Bailey, M. E., Sattar, N. \& Gill, J. M. 2013. Should physical activity recommendations be ethnicity-specific? Evidence from a cross-sectional study of South Asian and European men. PLoS ONE, 8, (12).

Coetzer, P., Noakes, T. D., Sanders, B., Lambert, M. I., Bosch, A. N., Wiggins, T. \& Dennis, S. C. 1993. Superior fatigue resistance of elite black South African distance runners. $J$ Appl Physiol (1985), 75, (4), 1822-7.

Cohen, J. (1988). Statistical power analysis for the behavioral sciences (2nd ed.). Hillsdale, NJ: Lawrence Earlbaum Associates.

Cook, M. D., Heffernan, K. S., Ranadive, S., Woods, J. A. \& Fernhall, B. 2013. Effect of resistance training on biomarkers of vascular function and oxidative stress in young African-American and Caucasian men. J Hum Hypertens, 27, (6), 388-92.

Cormie, P., Mcguigan, M. R. \& Newton, R. U. 2011. Developing maximal neuromuscular power: Part 1--biological basis of maximal power production. Sports Med, 41, (1), 1738.

Cubbon, R. M., Murgatroyd, S. R., Ferguson, C., Bowen, T. S., Rakobowchuk, M., Baliga, V., Cannon, D., Rajwani, A., Abbas, A., Kahn, M., Birch, K. M., Porter, K. E., Wheatcroft, S. B., Rossiter, H. B. \& Kearney, M. T. 2010. Human exercise-induced circulating 
progenitor cell mobilization is nitric oxide-dependent and is blunted in South Asian men. Arterioscler Thromb Vasc Biol, 30, (4), 878-84.

Eynon, N., Hanson, E. D., Lucia, A., Houweling, P. J., Garton, F., North, K. N. \& Bishop, D. J. 2013. Genes for elite power and sprint performance: ACTN3 leads the way. Sports Med, 43, (9), 803-17.

Fields, D. A., Bemben, M. G. \& Mayhew, J. L. 1997. The influence of muscle group location and race on the relationship between muscle strength and power. J Sports Med Phys Fitness, 37, (1), 24-31.

Folland, J. P. \& Williams, A. G. 2007. The adaptations to strength training : morphological and neurological contributions to increased strength. Sports Med, 37, (2), 145-68.

Hall, L. M., Moran, C. N., Milne, G. R., Wilson, J., Macfarlane, N. G., Forouhi, N. G., Hariharan, N., Salt, I. P., Sattar, N. \& Gill, J. M. 2010. Fat oxidation, fitness and skeletal muscle expression of oxidative/lipid metabolism genes in South Asians: implications for insulin resistance? PLoS One, 5, (12), e14197.

Hameed, U. A., Manzar, D., Raza, S., Shareef, M. Y. \& Hussain, M. E. 2012. Resistance Training Leads to Clinically Meaningful Improvements in Control of Glycemia and Muscular Strength in Untrained Middle-aged Patients with type 2 Diabetes Mellitus. $N$ Am J Med Sci, 4, (8), 336-43.

Knox, A., Sculthorpe, N. \& Grace, F. Short-term resistance training improves cardio-metabolic health in Caucasian and South Asian males. Poster presented at: American College of Sport Medicine World Congress on Exercise is Medicine; May 2015, Boston, MA.

Misra, A., Alappan, N. K., Vikram, N. K., Goel, K., Gupta, N., Mittal, K., Bhatt, S. \& Luthra, K. 2008. Effect of supervised progressive resistance-exercise training protocol on insulin sensitivity, glycemia, lipids, and body composition in Asian Indians with type 2 diabetes. Diabetes Care, 31, (7), 1282-7. 
167 Moritani, T. \& Devries, H. A. 1979. Neural factors versus hypertrophy in the time course of muscle strength gain. Am J Phys Med, 58, (3), 115-30.

169 Swift, D. L., Johannsen, N. M., Lavie, C. J., Earnest, C. P., Johnson, W. D., Blair, S. N.,

170 Church, T. S. \& Newton, R. L., Jr. 2013. Racial differences in the response of cardiorespiratory fitness to aerobic exercise training in Caucasian and African American postmenopausal women. J Appl Physiol (1985), 114, (10), 1375-82.

173 Walts, C. T., Hanson, E. D., Delmonico, M. J., Yao, L., Wang, M. Q. \& Hurley, B. F. 2008. 174 Do sex or race differences influence strength training effects on muscle or fat? Med Sci Sports Exerc, 40, (4), 669-76.

176 
177 Table legend

178

179 Table 1 - Exercise session design utilised during the resistance training intervention. Both

180 sessions involve the main compound exercises and accessory exercises.

181 Table 2 - Group mean data for initial training loads. These loads were prescribed in relation to

182 participant ability and not by a percentage of strength testing. This was to emphasise correct

183 technique and ensure overall safety of the participant. Data are presented as mean \pm standard 184 deviation (SD.).

185 Table 3- Descriptive characteristics of Caucasian (CAUC) and South Asian (SOU)

186 participants before (PRE) and after (POST) a 6-week resistance training programme. Session

187 A and B data display the group mean of the accumulated loads lifted of each exercise within

188 each session. Individual exercise data were derived by calculating group means of the loads

189 lifted for each exercise. Data are presented as group mean \pm SD. ${ }^{\varphi}$ - significant difference

190 between groups at same time point. $\Omega$ - significant difference within groups PRE to POST. *

$191-P<0.05, * *-P<0.01$.

192

193 Figure legends

194 Figure 1 - Lower body strength in Caucasians and South Asians before (PRE) and after

195 (POST) resistance training. Data are presented as group mean \pm SD). No significant

196 differences were observed at PRE $(P=0.454)$. A significant difference $(*)$ was apparent at

197 POST between groups $(P=0.013)$.

198 Figure 2 - Upper body strength in Caucasians and South Asians before (PRE) and following

199 resistance training (POST). Data are presented as group mean \pm SD). There were no

200 significant differences PRE $(P=0.727)$ or POST $(P=0.938)$ intervention.

201 Figure 3 - Progression of the squat exercise in Caucasians and South Asians during 6 weeks 202 of resistance training; data presented as group mean with standard deviations (mean \pm SD). A 
203 significant group $\times$ time interaction $(P<0.001)$ identified divergence in squat progression from

204 session 7 through to session 18 (completion). Main effects of time were observed in the CAUC 205 ( $\mathrm{P}<0.001)$ and the SOU $(\mathrm{P}<0.001)$ group. 